15

\title{
Применение искусственной нейронной сети в экспериментах по ядерному спиновому эху
}

\author{
() С.Н. Полулях, А.И. Горбованов \\ Крымский фредеральный университет им. В.И. Вернадского, Симферополь, Россия \\ E-mail: sergey.polulyakh@cfuv.ru
}

Поступило в Редакцию 15 февраля 2019 г.

В окончательной редакции 15 февраля 2019 г.

Принято к публикации 21 марта 2019 г.

\begin{abstract}
Продемонстрирована возможность использования искусственной нейронной сети для выделения сигналов ядерного спинового эха в условиях, когда амплитуда сигнала эха сравнима с амплитудой шума. Для обучения нейронной сети предложено использовать данные, полученные путем наложения модельных сигналов эха гауссовой формы на экспериментально зарегистрированные сигналы шумов.
\end{abstract}

Ключевые слова: ядерный магнитный резонанс, спиновое эхо, отношение сигнал-шум, искусственная нейронная сеть.

DOI: 10.21883/PJTF.2019.12.47911.17736

В экспериментах по регистрации импульсных откликов ядерного магнитного резонанса (ЯМР), в частности сигналов спинового эха, возникает проблема выделения сигнала на фоне сильных шумов. Для решения этой проблемы наряду с применением различного рода частотных фильтров используется накопление сигнала. Накопление осуществляется путем многократной регистрации импульсного отклика спиновой системы при одних и тех же условиях. При этом минимальный период повторения ограничен временем продольной релаксации ядерной намагниченности, что приводит к существенному увеличению времени эксперимента. С другой стороны, опытный экспериментатор может обнаружить сигнал эха в условиях сильных шумов даже при однократной регистрации импульсного отклика.

Целью настоящей работы является проверка возможности обучения искусственной нейронной сети (ИНС) выделять сигналы спинового эха при амплитудах сигнала, сравнимых с амплитудой шума. Основанием послужили успешные примеры применения ИНС для фильтрации и обработки шумов в речевых сигналах [1], коммуникационных сигналах, сигналах радаров и сонаров [2], электрокардиограммах [3], цифровых изображениях [4] и ряде других случаев.

Использовались экспериментально наблюдаемые сигналы двухимпульсного спинового эха от ядер ${ }^{57} \mathrm{Fe}$ в ферромагнитной пленке железо-иттриевого граната $\mathrm{Y}_{3} \mathrm{Fe}_{5} \mathrm{O}_{12}$ толщиной $22 \mu \mathrm{m}$, синтезированной методом жидкофазной эпитаксии на подложке из гадолиний-галлиевого граната, ориентированной в плоскости (111). Исследуемый образец обогащен магнитным изотопом ${ }^{57} \mathrm{Fe}$ до 96\%. Эксперименты выполнялись при комнатной температуре в нулевом внешнем магнитном поле.

Для формирования сигналов эха использовалось два возбуждающих импульса длительностями 2 и $4 \mu \mathrm{s}$, разделенных временны́м интервалом длительностью $100 \mu \mathrm{s}$.
Частота колебаний переменного магнитного поля во время действия импульсов составляла $54.1 \mathrm{MHz}$, что соответствует резонансу ядер ${ }^{57} \mathrm{Fe}$ тетраэдрических ионов $\mathrm{Fe}^{3+}[5]$.

Для регистрации использовалось амплитудное детектирование сигнала. Регистрация сигнала начиналась через $125 \mu$ s после первого возбуждающего импульса. Общее время регистрации отклика для одиночной возбуждающей последовательности импульсов составляло $512 \mu \mathrm{s}$. Для цифровой регистрации сигнала использован аналого-цифровой преобразователь с разрядностью 10 bit и временны́м шагом $0.5 \mu \mathrm{s}$. В результате одиночный скан состоял из 1024 точек. При выбранных временны́х интервалах можно было полагать, что начиная со 128-й микросекунды (последние 768 точек) регистрировались чистые шумы. В качестве примера на рисунке пунктирными линиями показаны первые 256 значений амплитуды зарегистрированного отклика. Сигналы на частях $a$ и $c$ рисунка получены при различных амплитудах возбуждающих импульсов и усилении приемника ЯМР-спектрометра. Данные, приведенные на рисунке, $e$, получены в отсутствие возбуждающих импульсов, когда появление сигнала эха не ожидается. Малость амплитуды эха обусловлена как малым объемом образца, так и выбранным временны́м интервалом между возбуждающими импульсами (время поперечной релаксации ядерной намагниченности составляет порядка $250 \mu \mathrm{s}[5])$.

Для обработки экспериментально зарегистрированных сигналов использована глубокая нейронная сеть, состоящая из трех слоев. Каждый нейрон ИНС имеет $N$ входов и один выход. Связь между сигналом на выходе нейрона $U$ и набором входных сигналов $u_{i}$ определяется функцией активации $f$

$$
U=f\left(\Sigma_{i=1}^{N} w_{i} u_{i}+b\right),
$$

где $u_{i}$ - величина сигнала на $i$-м входе нейрона, $w_{i}$ весовой множитель для $i$-го входа, $b$ - смещение, 

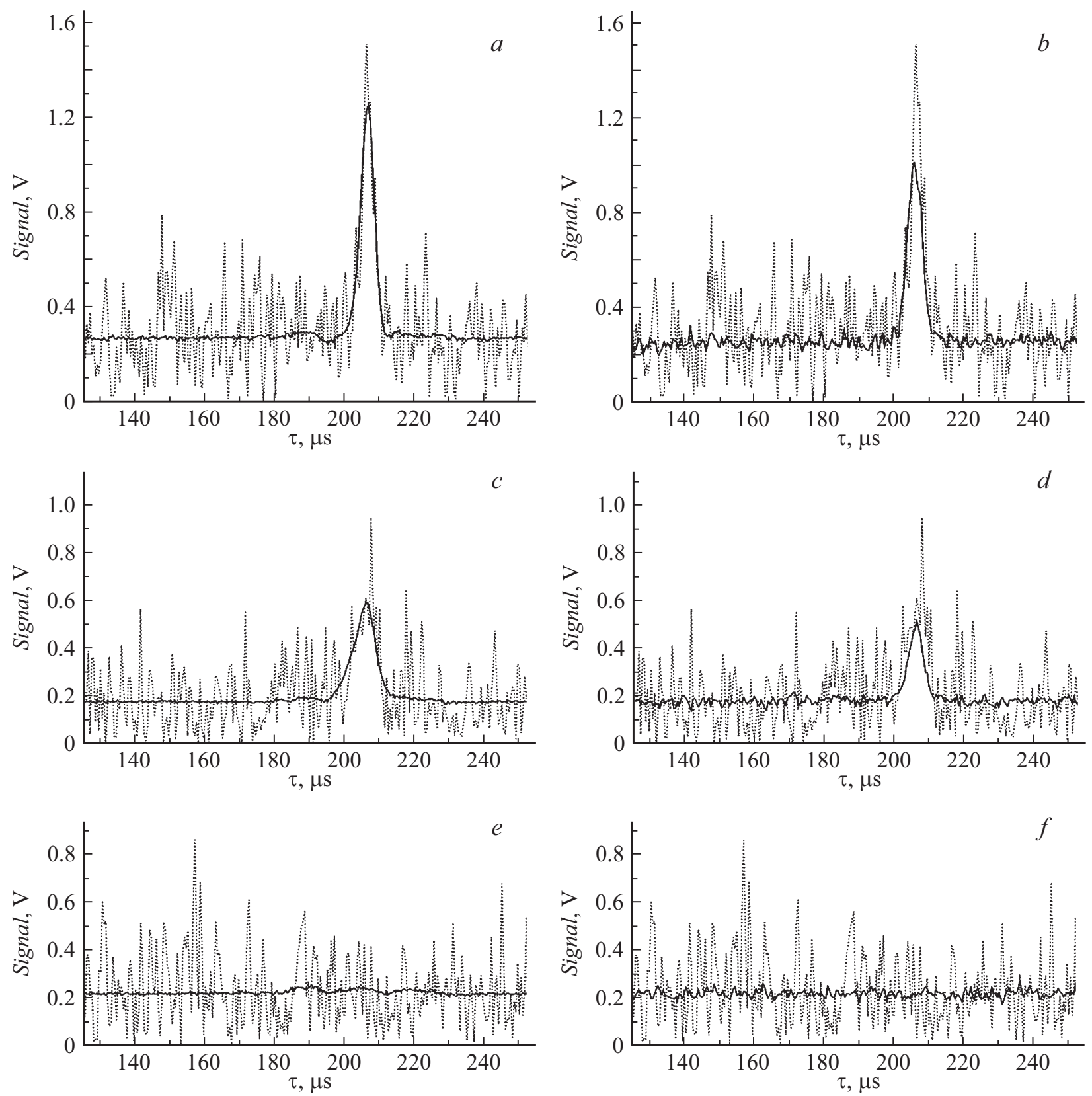

Экспериментально зарегистрированные сигналы при однократной регистрации (пунктирная линия). Сплошная линия - результат обработки сигнала искусственной нейронной сетью $(a, c, e)$ и результат усреднения по 100 сигналам, полученным при одних и тех же условиях $(b, d, f)$.

$f$ - функция активации. В частности, для функции активации ReLU (rectified linear unit) $f(x)=x$ при $x>0$ и $f(x)=0$ при $x \leq 0[6]$. В случае линейной функции активации (linear) $f(x)=x$ при любых $x$.

Первый слой использованной ИНС состоял из 1024 нейронов с функцией активации ReLU. Значения амплитуды входного сигнала в каждой из 256 точек подавались на вход каждого нейрона первого слоя. Второй (глубокий) слой также состоял из 1024 нейронов типа ReLU. Выходные сигналы каждого из нейронов первого слоя подавались на входы каждого из нейронов второго слоя. Последний слой сети состоял из 256 нейронов с линейной функцией активации. На входы каждого из нейронов последнего слоя подавались выходные сигналы каждого нейрона второго слоя. Искусственная нейронная сеть реализована на языке Python с использованием библиотек Tensorflow и Keras [7].

Обучение нейронной сети состоит в подборе весовых множителей $w_{i}$ и смещений $b$ каждого из нейронов. Для этого в настоящей работе использовалось „обучение с учителем“ методом обратного распространения ошибки, реализованным в Tensorflow. Основная задача состояла в подготовке данных для обучения сети. Каждый элемент набора данных для обучения (строка данных) содержит 
набор входных значений и данные разметки - правильный результат, который ожидается на выходе сети для данного набора входных значений.

При подготовке данных для обучения полагалось, что сигнал на входе сети представляет собой сумму сигнала ядерной индукции (спиновое эхо в нашем случае) и шума. Один элемент набора данных представлял собой 256 значений экспериментально зарегистрированной амплитуды шума (одиночный скан), к которым прибавлялось модельное эхо гауссовой формы. Значение амплитуды $A_{i}$ в $i$-й точке рассчитывалось с помощью выражения

$$
A_{i}=A_{0} \exp \left(-\frac{(i-k)^{2}}{2 \sigma^{2}}\right)
$$

В качестве данных разметки использовались те же самые 256 значений амплитуды сигнала, которые были рассчитаны с помощью выражения (2) для подготовки входных значений. Всего было подготовлено 6500 последовательностей на основе экспериментально зарегистрированных шумов. Одна и та же реализация шумового процесса использовалась только один раз. Параметры модельного сигнала (2) для каждой из последовательностей определялись с помощью генератора псевдослучайных чисел и лежали в следующих пределах: для амплитуды сигнала $A_{0}=0.10-1.35$, для дисперсии $\sigma=3.0-15.0$ и для номера входа, соответствующего максимуму сигнала, $k=100-200$. При обучении сети для функции потерь использовалось среднеквадратичное отклонение (loss='mean_squared_error' ${ }^{\prime}$ ).

Сеть, обученная на сигналах, представляющих собой сумму модельного сигнала эха (2) и экспериментально зарегистрированного шума, использовалась для обработки экспериментально наблюдаемых сигналов эха в условиях сильного шума. В качестве примера на рисунке сплошными линиями показаны сигналы, полученные на выходе ИНС (зависимости на частях $a, c$ и $e$ ). На вход сети подавался сигнал, полученный в результате однократной регистрации импульсного отклика (показан пунктирной линией). Результат усреднения при 100-кратном накоплении представлен сплошными линиями на частях $b, d$ и $c$ рисунка (пунктирная линия повторяет результат однократной регистрации). Данные, приведенные на части $b$ рисунка, получены при тех же условиях, что и данные, представленные на части $a$ (аналогично для зависимостей на частях $c, d$ и $e, f$ ).

Результаты, приведенные на рисунке, показывают, что ИНС способна при одиночном скане выделить сигнал эха, амплитуда которого сравнима с амплитудой шума. Фактически процедура накопления может быть выполнена один раз, на этапе обучения ИНС. Специальным образом обученная ИНС может быть использована в дальнейшем для выделения сигнала на фоне сильных шумов при однократной регистрации сигнала. Для демонстрации возможностей ИНС в настоящей работе использована довольно простая трехслойная сеть. Дальнейшее развитие предлагаемого подхода может состоять, в частности, в использовании ИНС более сложной архитектуры.

\section{Финансирование работы}

Исследование выполнено при финансовой поддержке Российского фонда фундаментальных исследований в рамках научного проекта № 18-29-02120.

\section{Конфликт интересов}

Авторы заявляют, что у них нет конфликта интересов.

\section{Список литературы}

[1] Yin S., Liu C., Zhang Z., Lin Y., Wang D., Tejedor J., Zheng T.F., Li Y. // EURASIP J. Audio Speech Music Proc. 2015. N 1. P. 2 (1-14). DOI: 10.1186/s13636-014-0047-0

[2] Khairnar D.G., Merchant S.N., Desai U.B. // J. Comput. 2008. V. 3. N 1. P. 32-39.

[3] John J.N., Galloway C., Valys A. Deep convolutional neural networks for noise detection in ECGs. arXiv: $1810.04122 \mathrm{v} 1.2018$

[4] Zhang F., Cai N., Wu J., Cen G., Wang H., Chen X. // IET Image Proc. 2018. V. 12. N 4. P. 485-493.

DOI: 10.1049/iet-ipr.2017.0389

[5] Бержанский В.Н., Полулях С.Н. // ФТТ. 1989. Т. 31. В. 8. C. $256-258$.

[6] Nair V., Hinton G.E. Rectified linear units improve restricted boltzmann machines // 27th Int. Conf. on machine learning. Madison, USA: Omnipress, 2010. P. 807-814.

[7] https://keras.io 